\title{
A QoE Performance Evaluation Framework for LTE Networks
}

\author{
D. Ciambrone*, S. Tennina*, D. Tsolkas ${ }^{\dagger}$ and L. Pomante \\ *WEST Aquila S.r.l., L'Aquila, Italy \\ \{daniele.ciambrone, tennina\} at westaquila.com \\ $\dagger^{\dagger}$ Department of Informatics and Telecommunications, University of Athens, Greece, \\ dtsolkas at di.uoa.gr \\ $\ddagger$ Center of Excellence DEWS, University of L’Aquila, Italy \\ luigi.pomante at univaq.it
}

\begin{abstract}
The CASPER project envisions the user-centric network and service management in future mobile networks. In this frame, this paper aims at studying the impact of packet loss rate (PLR) and end-to-end delay (E2E delay) induced by quality of experience (QoE)-aware management policies in real LTE networking conditions with Voice over IP and video streaming traffic patterns. This objective is achieved by implementing a simulation framework built upon SimuLTE/OMNeT++ to reproduce the CASPER reference scenarios. The results demonstrate that there is a clear trade-off between the PLR and E2E delay that needs to be taken into account to keep acceptable QoE levels.

Index Terms-Quality of Experience, VoIP, LTE, System Level Simulation, Packet Loss Rate, End-to-End Delay.
\end{abstract}

\section{INTRODUCTION}

Quality of Experience (QoE) is a recently proposed acronym to evolve from the well-known Quality of Service (QoS) to express the perception of the quality of both network and service from the end-user perspective [1]. QoE quantifies end-user's (e.g., video consumer's) satisfaction level. It is indirectly correlated with user's engagement with the service and is a crucial factor for enabling advanced customer experience management (CEM). The primary objective of CASPER project [2] is to combine academic and industrial forces towards leveraging the expected benefits of QoE exploitation in 5G networks.

CASPER aims at the definition of an integrated middleware architecture, following industrial requirements and recent standardisation activity. The user-centric logic of the CASPER project is based on an in-depth QoE analysis and adopts mainly results from the available literature (e.g., [3]), combined with the flexibility and reconfigurability provided by the software-defined networking (SDN) [4] and network function virtualisation (NFV) [5] technologies.

For a beneficial intervention of QoE in network and service management in the $5 \mathrm{G}$ era three interlinked modules are proposed [6]: (i) Reliable and passive QoE monitoring, including adaptive and objective QoE estimation mechanisms; (ii) Robust and real-time QoE-driven service management and CEM policies; (iii) Efficient QoE controller to realise the interface between the middleware and the network functions.

In this paper the objective is to present our system level simulator built upon SimuLTE/OMNeT++ [7], [8] to repro-

978-1-5386-4725-7/18/\$31.00 @2018 IEEE

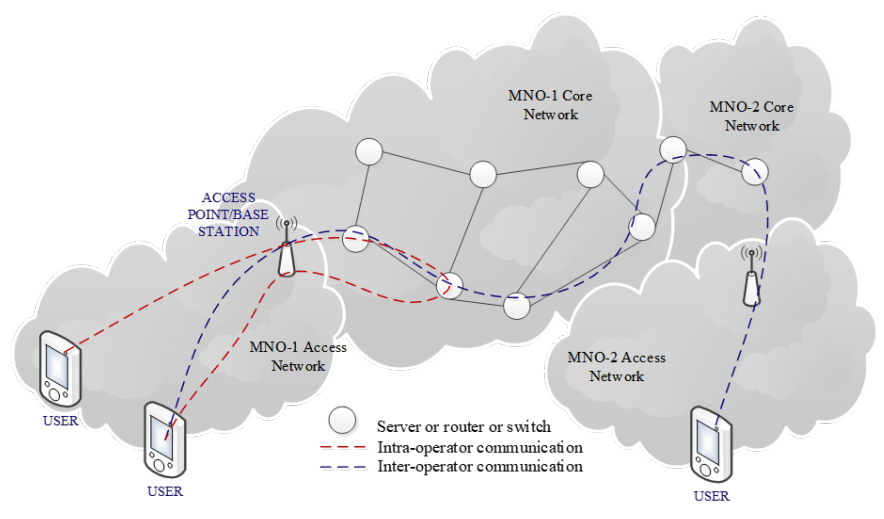

Fig. 1. Scenario A - Intra- or inter- operator voice call.

duce the CASPER reference scenarios and evaluate the effects of monitoring and control (i.e., management) policies over the final QoE perceived by the end users.

Consequently, the remainder of the paper is organised as follows. Section II recalls the scenarios addressed in the CASPER project, as previously introduced in [2], while Section III presents the implementation we did of those scenarios into the simulation framework and how we simulated the effects of QoE management policies. After describing the simulation setup, Section IV discusses the impact of the management policy on the QoE and shows the trade-off between the endto-end delay and the packet loss rate for VoIP services. Finally, Section V concludes the paper.

\section{ScEnARIo Definitions}

\section{A. Scenario A: Real-time Communication between Two Par-} ties

The first scenario includes any real-time communication between two parties, directly via the infrastructure of a Mobile Network Operator (MNO) or Internet Service Provider (ISP). The involved users have connectivity to the same or different operators (intra-operator and inter-operator cases, respectively) and they may be located in the same or different cells. This scenario is presented in Fig. 1.

The main application to be studied here is Voice over IP (VoIP), but also Short Message Service (SMS) or video 


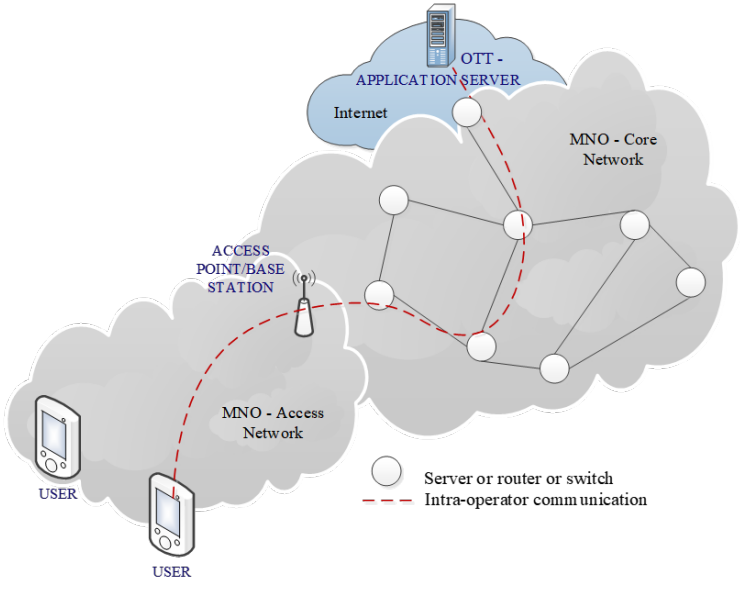

Fig. 2. Scenario B - video streaming/Online video consumption.

calls fall into the same scenario. A significant challenge in this scenario is the comprehension of QoE Key Performance Indicators (KPIs) for voice calls, as well as the guaranteeing of excellent QoE under challenging network conditions or high congestion. Moreover, if multiple MNO/ISPs are involved, issues arise regarding liability in case of low QoE.

\section{B. Scenario B: Multimedia Content Delivery from a Service Provider}

This scenario mainly concerns cases of video content delivery, namely video streaming. The common paradigm nowadays is that the Over The Top (OTT) parties provide access to video streaming services, that the users can access only through their MNO or ISP connection. (Note that MNOs or ISPs may also provide video streaming services by themselves to their subscribers.) The second scenario of CASPER is presented in Fig. 2.

The main applications that are going to be studied here are: (i) Non-adaptive video streaming (non-real-time service). (ii) HTTP Adaptive Streaming (non-real-time service). (iii) Live TV / IPTV (real-time service).

The major challenges in this category of scenarios stem from the fact that the video content is usually made available by an OTT provider, whereas it is delivered by a traditional MNO/ISP. Therefore, QoS/QoE guarantees from the OTT side are mostly absent and are mainly oriented to providing highresolution video content to the viewers. However, the delivery procedure of the content itself is at the discretion of the MNO/ISP. Consequently, if the quality of the offered video is bad despite the OTT's proactive measures, a closer interaction between MNO/ISP and OTT is needed to improve the system's efficiency from the end user perspective.

\section{Scenario C: Real-time Communication among Multiple Parties via an Intermediate Server}

The third category of scenarios is the most challenging one since it includes an OTT provider, one or more MNO/ISP providers and multiple users. Applications that fall into this category are Skype calls (voice or video), Viber, gaming,

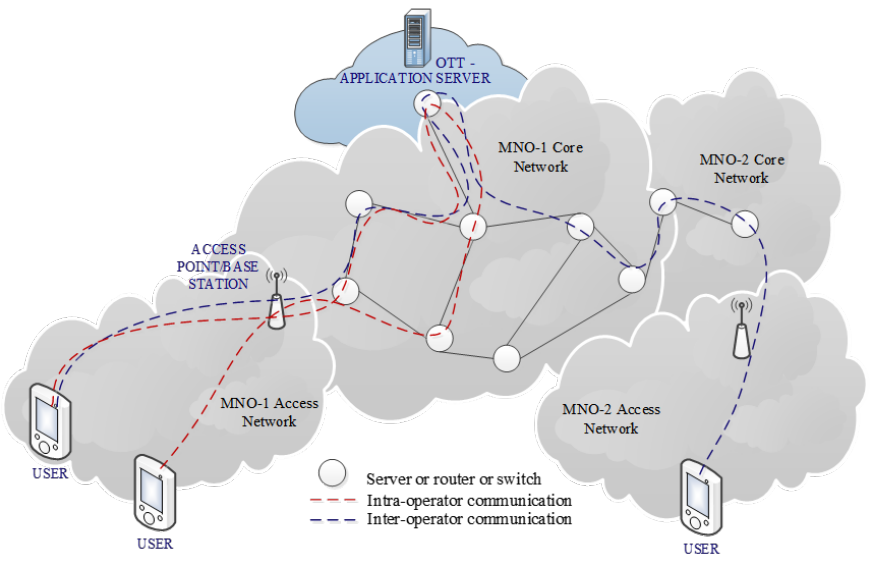

Fig. 3. Scenario C - Skype call or web conference.

and teleconference, among others. The traffic, in this case, is accumulated at a server in possession of the OTT party, passing however through the network infrastructure of one or more MNO/ISPs (intra-operator and inter-operator case, respectively). This scenario is presented in Fig. 3.

The challenges, in this case, include the ones discussed in the previous two scenarios, as well as the fact that this one involves multimedia content delivery. A major challenging issue from a QoE perspective is also that proper and reliable QoE estimation models for these types of applications are not available yet, and finding appropriate KPIs is not a trivial issue. The CASPER architecture will tackle these challenges by providing interfaces to all involved parties for QoE monitoring as well as control. It will, therefore, serve as a platform that enables interactions among the different stakeholders, with the target of providing user-personalised, application-aware and context-aware QoE improvements.

\section{IMPLEMENTATION AND SimUlation}

The simulation tool chosen to reproduce the CASPER scenarios, as recalled in the previous section, is SimuLTE [7], which allows reproducing LTE-based wireless and wired network architectures, building on the INET Framework and the OMNeT++ discrete event simulator [8].

To implement the scenarios in the simulation environment, we opted to start from the third one, which is the most complete. As recalled, this scenario mainly concerns VoIPbased traffic between two user equipment $(U E s)$, over a single or multiple MNO/ISP providers and passing through an OTTserver. The network topology is shown in Fig. 4.

We have developed a new module at the application layer, starting from the existing VoIP Application defined in the INET library. The basic application is composed of a VoIP Sender and a VoIP Receiver, to send and receive VoIP packets between $U E s$, respectively. VoIP traffic is composed of a certain number of talks, each one has a variable number of packets to send, following a Weibull random distribution [9]. The sender application generates the packets, increase the talks' ID and sends them, while the receiver application's duty 


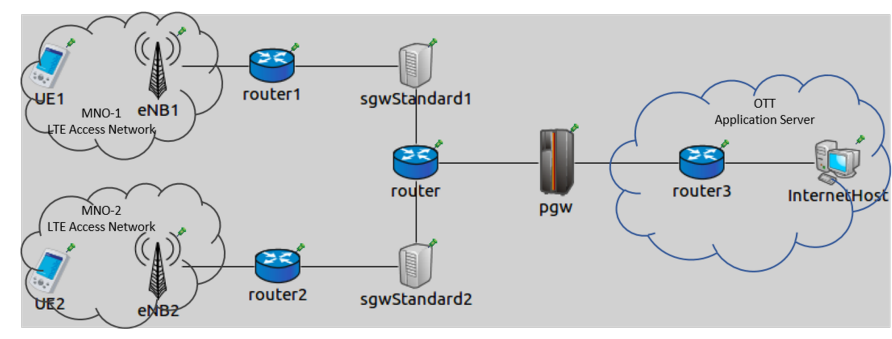

Fig. 4. Implementation of the scenario $\mathrm{C}$ of Fig. 3 in the chosen simulation environment.

mainly lies in the computation of statistics. These applications have been extended to implement a new module for the OTT provider, i.e., the VoIP Server, which combines the features of VoIP Sender and VoIP Receiver, to receive VoIP packets from the source and forward to the destination.

In the simple approach of the static network topology sketched in Fig. 4, simulations execute in ideal conditions, where packet loss rates and delays are always low and mainly depends on the propagation conditions in the wireless access portion of the LTE architecture. However, in the real world, network congestions and other impairments happen, which affects the packet loss rate and the end-to-end delay. The objective is to control the simulation parameters so that the evidence about the tradeoff of packet loss rate and end-toend delay for the perceived QoE at the end-users is given. To do this, we imagine that at a given time $t=T>0$ an impairment arises in the network: a QoE monitoring entity recognises it and starts taking the QoE control actions, e.g., by instructing the MNO/ISP to route packets on a different path. Then impairment takes resolved at time $t=T+D$, where $D>0$. In our view, the network impairment and the action of the QoE management policy are taken into account as an increased packet loss rate and an additional end-to-end delay, during the interval $t \in[T, T+D]$.

In other analytical-oriented approaches, the effects of the packet loss rate and the end-to-end delay on the QoE might result from numerical simulations of any given model of the underlying network. It is worth stressing that in this paper, the added value is that the simulations run over the full protocol stack of an LTE network architecture, and just the additional PLR and E2E delay are modelled as described, to take into account the effects of any QoE management policy.

Without loss of generality, such increases are taken into account by implementing a middleware layer as shown in Fig. 5. More precisely, the application modules VoIP sender or VoIP receiver together with this middleware constitute a new compound module [10] at the application layer interfaced with the transport layer of the underlying INET/SimuLTE protocol stack.

With this implementation, the simulation for the reference scenario proceeds as sketched in Fig. 6 and described next. After the initialisation of the network, the simulation starts. The VoIPSender application at $U E 1$ generates VoIP packets to be delivered to the OTT server (i.e., Internet Host). It then forwards all traffic to the $U E 2$, which receives at the

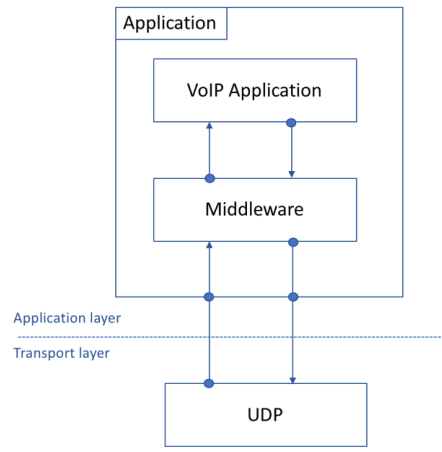

Fig. 5. Application and Middleware layers on top of the INET protocol stack.

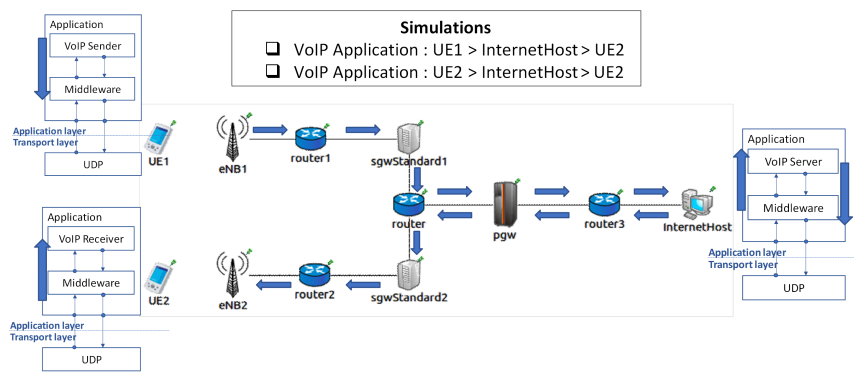

Fig. 6. Implementation of Scenario $\mathrm{C}$ as in Fig. 3.

VoIPReceiver application. For repeatability of the simulations under different conditions, in this scenario, the VoIP traffic is routed through different routers adopting static routing policies. Same applies for SGWs and PGW that forward traffic through static routes. In particular, PGW implements a filter table to direct traffic differentiating source and destination through identifiers. Apparently, a different set of simulations can run adopting the reverse direction of the traffic flow, (i.e., from $U E 2$ to $U E 1$ ) or adding other $U E s$ (i.e., parallel and simultaneous flows).

In terms of simulated network topology, the other scenarios described in Section II can be derived from Fig. 6, since they just use a sub-network of the whole network. More in details, Fig. 7 shows the implementation of the second scenario, which refers to Fig. 2. In this scenario, a video streaming service runs at the application layers of $10 \mathrm{UEs}$ and the OTT server. Each $U E$ sends first a stream request to the server, and then the server starts to process the video.

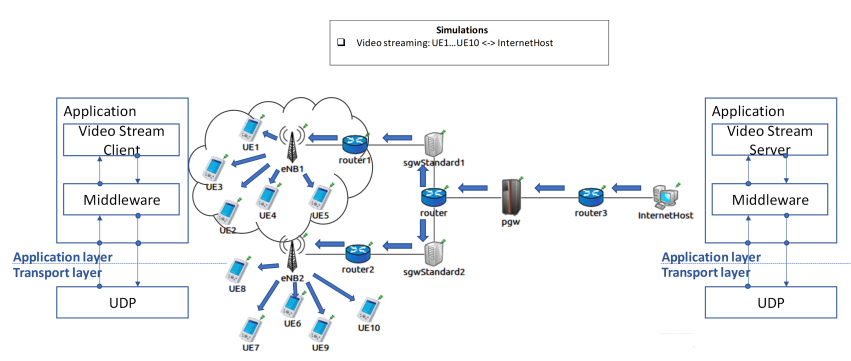

Fig. 7. Implementation of Scenario B as in Fig. 2. 


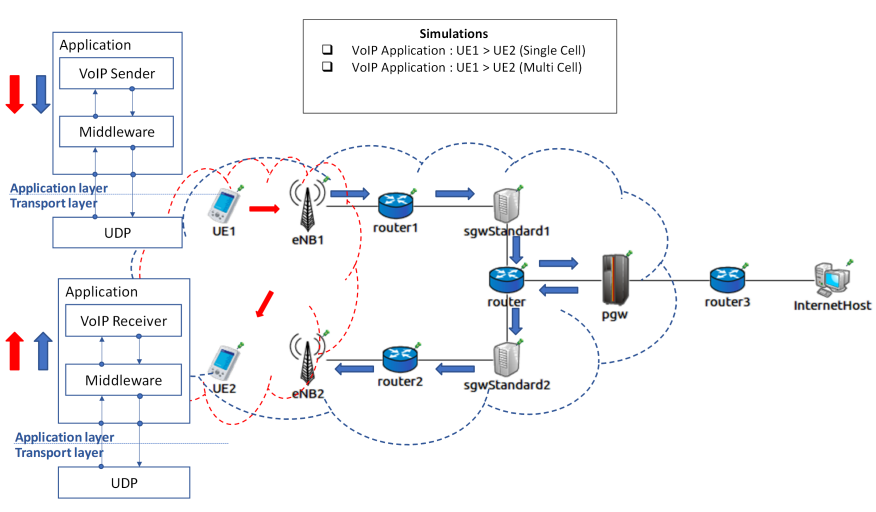

Fig. 8. Implementation of Scenario A as in Fig. 1.

The download can be executed simultaneously by one or more users or in different periods. The traffic is modelled through a specific data structure, defining the video size, the packet size and the packets' send interval. Similarly to the previous case of VoIP, the modules VideoStreamCli and VideoStreamSvr together with the middleware constitute the application layer of the streaming service, and generate the statistics. Finally, the last scenario is sketched in Fig. 8, which represents a realtime communication flow between $U E s$, in intra-operator and inter-operator cases, where the OTT server is not involved.

\section{Simulation Setup and Results}

\section{A. Simulation Setup}

In this section, we analyse the main metrics and specifications related to the simulations to obtain results and realise statistics. For the sake of simplicity, we report here the results of the simulations in the Scenario C of Fig. 3, as the most complete of elements, and the Scenario B of Fig. 2, while the last CASPER scenario will be further investigated in our on-going and future work. Simulation setup is finalised to study the primary QoE metrics for VoIP and Streaming traffic: Packet Loss Rate $(P L R)$ and End-to-End Delay $(E 2 E)$, while other network results can be evaluated for different application scenarios, including, e.g., Throughput and Jitter.

In our simulated world, about the Scenario C, the statistics of $P L R$ and $E 2 E$ are implemented at the receiver side in the $\mathrm{C}++$ code of the Middleware module (Fig. 5), transparently to the VoIP Receiver module, where the resulting QoE rank is computed. More in detail, a set of values were defined for the $P \hat{L} R$, while it holds $E 2 E \sim \mathcal{N}(\mu, \sigma)$, i.e., the increased end-to-end delay follows a Gaussian Distribution whose average and standard deviation parameters varies as described next. Consequently, different simulations have been executed by the full set of combinations of: (i) $P \hat{L} R=\{2 \%, 5 \%, 10 \%, 30 \%, 50 \%, 75 \%\}$. (ii) $\mu=$ $\{0.1,0.2,0.3,0.4,0.5\}$ s. (iii) $\sigma=0.05 \mathrm{~s}$.

Each simulation constitutes of 500 VoIP talks, each one having a random number of packets, according to the Weibull distribution, which models talk and silence durations.

Similar considerations for the Scenario B. The statistics of $P L R$ are implemented at the client side in the
$\mathrm{C}++$ code of the Middleware module, transparently to the VideoStreamingCli module, where the resulting QoE rank is computed. Different simulations have been executed for $P \hat{L} R=\{2 \%, 5 \%, 10 \%, 30 \%, 50 \%, 75 \%\}$. In each simulation ten users download a video from the server and each run a different video is used. In the end, for each user at least 10000 packets flow through the network.

Our objective in this paper is to measure the QoE for the VoIP and Video Streaming services. Then, to quantify the QoE, we have adopted the E-model [11] and the ITU P.563 [12] for VoIP service and the ITU-T G.1070 [13] for Video Streaming service. Both models express the QoE ranks in terms of Mean Opinion Score (MOS), as previously illustrated in [14], and reported in the next subsection, for the sake of completeness.

\section{B. QoE Ranking Formulas}

About VoIP traffic, the E-model has been proposed by the ITU-T for objectively measuring the MOS of voice communications by estimating the mouth-to-ear conversational quality as perceived by the user at the receive side. It is a parametric model that takes into account a variety of transmission impairments producing the so-called Transmission Rating factor $(R$ factor) scaling from 0 (worst) to 100 (best). Then a mathematic formula is used to translate this to MOS values [14]. When delays and signal impairments are involved in a realistic scenario, the $R$ factor is given by:

$$
R=R_{0}-\left(I_{s}+I_{d}+I_{e f}\right)+A
$$

where: $R_{0}=94.2$ is the $R$ factor in the baseline scenario with no network nor equipment impairments; $I_{s}$ : the impairments that are generated during the voice travelling into the network; $I_{d}$ : the delays introduced from the end-to-end signal travelling; $I_{e f}$ : the impairments introduced by the equipment and also due to randomly distributed packet losses; $A$ : allows for compensation of impairment factors when there are other advantages of access to the user (Advantage Factor). It describes the tolerance of a user due to a certain advantage that he/she enjoys, e.g., not paying for the service or being mobile. Typical value for cellular networks is $A=10$. Focusing on parameters which depend on the wireless part of the communication (transmissions between (H)eNB and UEs) it holds that [15]:

$$
I_{d}=0.024 d+0.11(d-177.3) H(d-177.3)
$$

where

$$
H(x)= \begin{cases}0, & x<0 \\ 1, & x \geq 0\end{cases}
$$

and $d$ is the average packet delivery delay. Also, assuming that the codec G.729a [16] is used, the packet loss rate, referred here as $p$, affects the parameter $I_{e f}$ as follows [15]:

$$
I_{e f}=11+40 \ln (1+10 p)
$$

The factor can be used as an assessment value; however, we transform it to MOS values to retrieve results comparable with results provided by subjective methods. The transformation formula is: $M O S=1$, if $R<0 ; M O S=4.5$, if $R>100$ 
and $M O S=1+0.035 R+R(R-60)(100-R) \cdot 7 \cdot 10^{-6}$ otherwise.

About the Video Streaming service, ITU-T has proposed the model for measuring the MOS as by the following expression:

$$
M O S=1+I_{\text {coding }} \cdot I_{\text {transmission }}
$$

with

$$
I_{\text {coding }}=I_{O_{f r}} \exp \left\{-\frac{\left(\left(\ln \left(F_{R_{v}}\right)-\ln \left(O_{f r}\right)\right)^{2}\right.}{2 D F_{R_{v}}^{2}}\right\}
$$

representing the video quality affected by the coding distortion and

$$
I_{\text {transmission }}=\exp \left\{-\frac{P_{p l_{V}}}{D_{P_{p l_{V}}}}\right\}
$$

representing the video quality affected by the transmission process. If the video frame rate $F r_{v}$, the video bit rate $B r_{v}$ and the video packet loss rate $P_{p l_{V}}$ are known, the factors $O_{f r}, I_{O f r}, D F_{R v}$ and $D_{P_{p l} V}$ can be computed based on 12 coefficients as follows:

$$
\begin{aligned}
& O_{f r}=v_{1}+v_{2} B r_{v} \\
& I_{O_{f r}}=v_{3}-\frac{v_{3}}{1+\left(\frac{B_{r_{v}}}{v_{4}}\right)^{v_{5}}} \\
& D F_{R_{v}}=v_{6}+v_{7} B r_{v} \\
& D_{P_{p l_{V}}}=v_{10}+v_{11} \exp \left\{\frac{-F r_{v}}{v_{8}}\right\}+v_{12} \exp \left\{\frac{-B r_{v}}{v_{9}}\right\}
\end{aligned}
$$

These coefficients depend on codec type, video format, key frame interval and video display size. Their default values for specific configurations may be found in [13] or may be derived using a standard methodology, also provided in [13].

\section{QoE Results}

To try to determine the trade-off between the $P \hat{L} R$ and E2E delay and to give bounds within which any QoE management policy must be confined not to degrade the performance felt by the end-users, we have analysed the average MOS against each of the $P \hat{L} R$ and $\mu$ combinations in Fig. 9. Assuming to accept a lower limit of $M O S=3.5$ for the QoE that is considered as acceptable by end-users for the given application service (i.e., in this case, VoIP) there is a clear trade-off between the PLR and the average E2E Delay that any QoE management policy needs to take into account. For instance, from Fig. 9(b), we can claim that the system's working point should always be in the lower left area, where the average PLR is below 25\%, and the average E2E Delay is below $300 \mathrm{~ms}$.

Similarly for the video streaming application of Scenario B (Fig. 2), we have analyzed the MOS against the $P \hat{L} R$. In our simulations, we used two encoding options, i.e., MPEG4 and H.264, and the full combination of three values for Frame Rate and Bit Rate, i.e., $F r_{v}=\in[20,25,30]$ fps and $B r_{v} \in[500,5000,50000]$ kbit/s. As Fig. 10 shows, regardless of the coding options, the best achieved MOS suddenly degrades as the $P \hat{L} R$ increases above roughly $10 \%$ and for the lower bit rates it even continually keeps below the
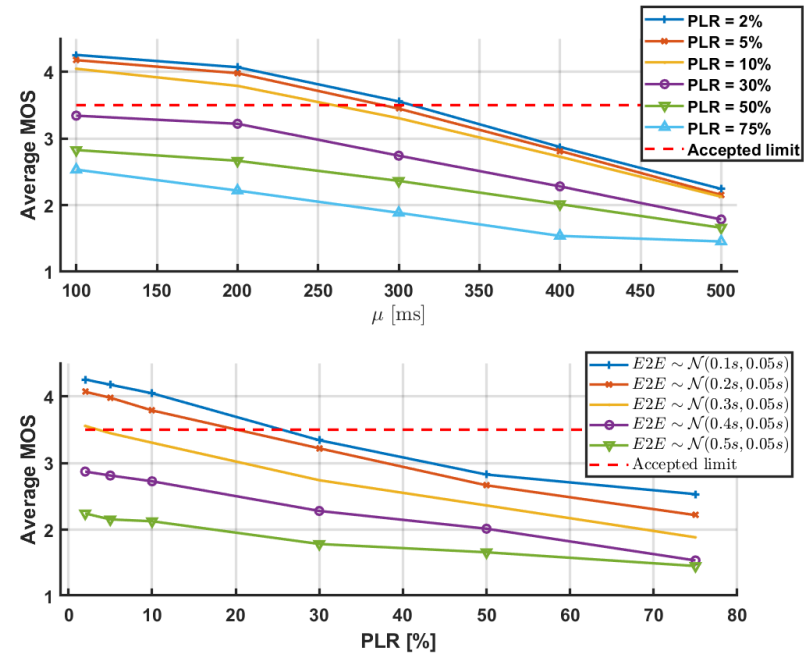

(a) Traces with the limit for the acceptable value of the average QoE/MOS.

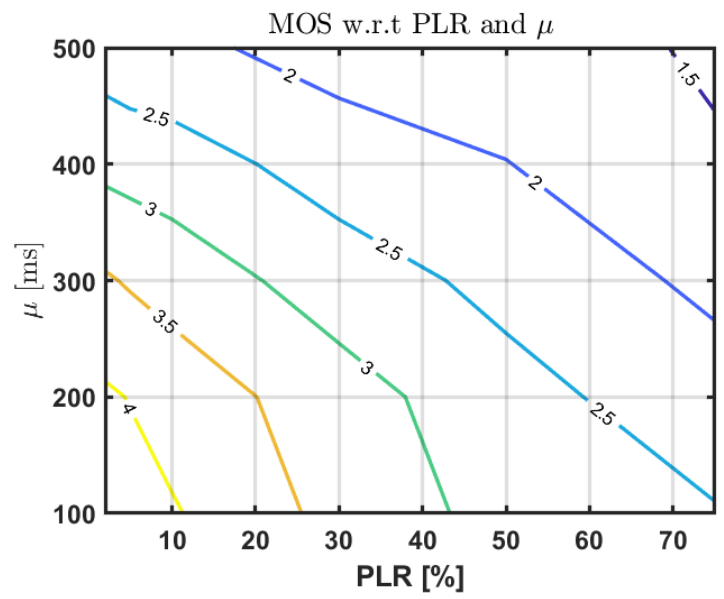

(b) Contour plot.

Fig. 9. Average QoE/MOS of VoIP of Scenario C (Fig. 3) against the combinations of $P \hat{L} R$ and $\mu$ for $\sigma=0.05 \mathrm{~s}$.

acceptable threshold. On the other hand, to better understand the differences between the different coding options, Fig. 11 shows the average MOS obtained for all the simulations with $P \hat{L} R<30 \%$. In this case, it is clear that the frame rate has a small impact on the average MOS, while it dramatically improves as the bit rate increases. Regarding the encoding algorithms, in the considered scenarios, the MPEG-4 slightly outperforms the H.264: this is dependent on the $v$ coefficients [13]. These results define where the working system point for the video streaming application scenarios should be. Moreover, these findings suggest that a QoE-driven adaptive streaming approach, to monitor the actual packet loss rate and adapt the bit rate of the encoded video, is the key for achieving an always high quality of experience.

\section{CONClusion}

In this paper, we have given an overview of the system level simulator proposed in the frame of CASPER project. More in 


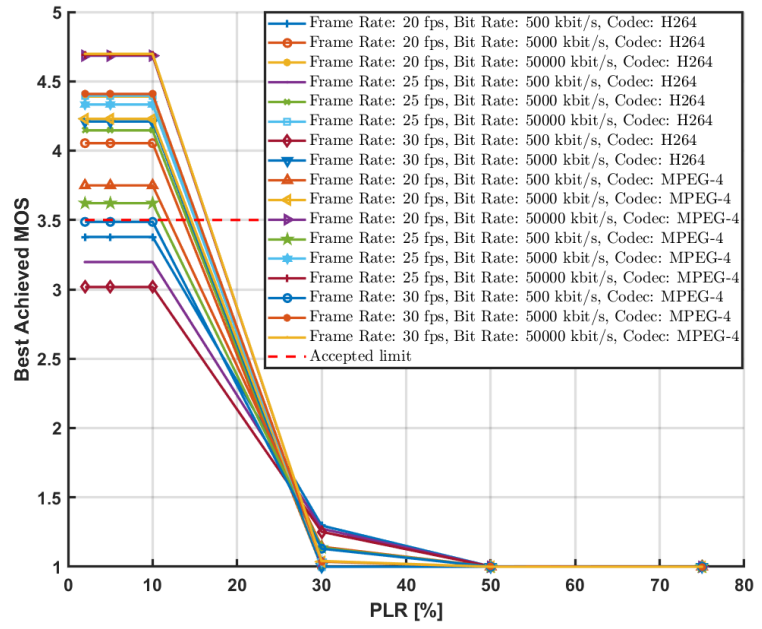

Fig. 10. Best achieved QoE/MOS for Video Streaming of Scenario B (Fig. 2) against the $P \hat{L} R$, for different video coding options.
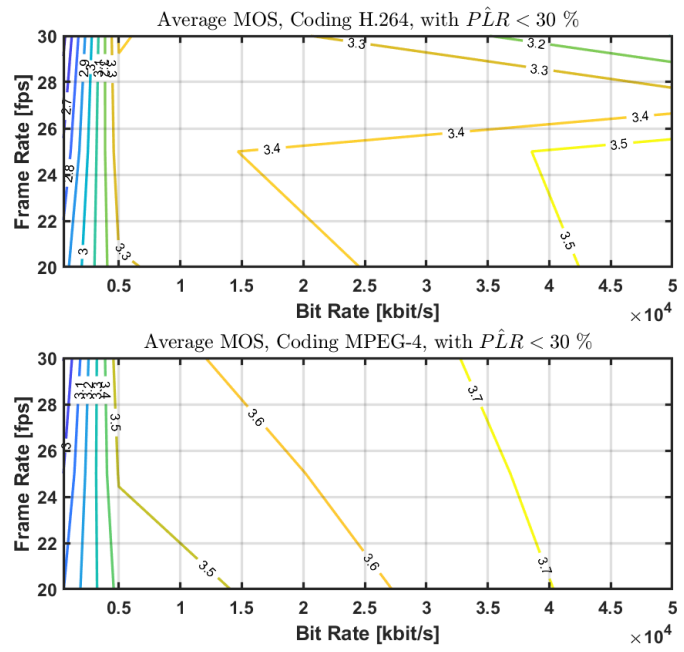

Fig. 11. Average QoE/MOS for Video Streaming of Scenario B (Fig. 2) for $P \hat{L} R<30 \%$ and different video coding options.

details, we recalled the reference scenarios addressed in the project, and we sketched how they are reproduced within the simulator. Then, preliminary performance analysis has been given for the VoIP application service in the third scenario and for the Video Streaming application service in the second scenario. This analysis demonstrated that appropriate QoE management policies must be designed taking into account that there is a trade-off between the end-to-end delay and the packet loss rate for the first service, and the packet loss rate only for the second service.

Our on-going work is revolving around the implementation of different applications than VoIP and video streaming, e.g., the HTTP Adaptive Streaming. Similarly, scaling up the simulation setups by including traffic flows from multiple parallel users and introducing more complex network topologies is another objective, to validate our findings in the full set of the CASPER scenarios.

\section{ACKNOWLEDGEMENT}

The authors would like to acknowledge the European Commission project CASPER (Grant number 645393), an H2020MSCA-RISE action. They also acknowledge Greek Financial Support from the State Scholarships Foundation (IKY) in the context of the action Excellence for Postdoctoral Researchers NSRF 1st Cycle for 2014-2020 (programme: Human Resources Development, Education and Lifelong learning).

\section{REFERENCES}

[1] R. Stankiewicz, P. Cholda, and A. Jajszczyk, "Qox: What is it really?" IEEE Communications Magazine, vol. 49, no. 4, pp. 148-158, April 2011.

[2] A. Colarieti, A. Marotta, M. Mpervarakis, L. Pomante, and V. Tsolkas, "QoE provisioning over mobile networks: The CASPER perspective," in 2017 IEEE 22nd International Workshop on Computer Aided Modeling and Design of Communication Links and Networks (CAMAD), June 2017, pp. 1-5.

[3] D. Tsolkas, E. Liotou, N. Passas, and L. Merakos, "A survey on parametric qoe estimation for popular services," J. Netw. Comput. Appl., vol. 77, no. C, pp. 1-17, Jan. 2017. [Online]. Available: https://doi.org/10.1016/j.jnca.2016.10.016

[4] O. Networking Foundation, "Software-defined networking: The new norm for networks," 042012.

[5] ETSI. (2014) Network functions virtualisation (NFV); architectural framework. [Online]. Available: https://portal.etsi.org/Portals/0/ TBpages/NFV/Docs/NFV_White_Paper3.pdf

[6] E. Liotou, A. Marotta, L. Pomante, and K. Ramantas, "A middleware architecture for qoe provisioning in mobile networks," in 2017 IEEE 22nd International Workshop on Computer Aided Modeling and Design of Communication Links and Networks (CAMAD), June 2017, pp. 1-5.

[7] A. Virdis, G. Stea, and G. Nardini, "SimuLTE - a modular system-level simulator for LTE/LTE-A networks based on OMNeT++," in 2014 4th International Conference On Simulation And Modeling Methodologies, Technologies And Applications (SIMULTECH), Aug 2014, pp. 59-70.

[8] Z. Bojthe, L. Meszaros, B. Seregi, R. Hornig, and A. Varga, INET Framework for OMNeT++, 2016.

[9] S. Guha and N. Daswani, "An experimental study of the skype peer-topeer voip system," Cornell University, Tech. Rep., 2005.

[10] A. Varga and R. Hornig, "An overview of the omnet++ simulation environment," in Proceedings of the 1st international conference on Simulation tools and techniques for communications, networks and systems \& workshops. ICST (Institute for Computer Sciences, SocialInformatics and Telecommunications Engineering), 2008, p. 60.

[11] J. A. Bergstra and C. Middelburg, "ITU-T recommendation g.107: The E-Model, a computational model for use in transmission planning," 2003.

[12] T. Falk and W. Chan, "Single ended method for objective speech quality assessment in narrowband telephony applications," ITU-T, p. 563, 2004.

[13] ITU-T, "Recommendation g.1070: Opinion model for video-telephony applications," 2012.

[14] D. Tsolkas, E. Liotou, N. Passas, and L. Merakos, "The need for qoedriven interference management in femtocell-overlaid cellular networks," in International Conference on Mobile and Ubiquitous Systems: Computing, Networking, and Services. Springer, 2013, pp. 588-601.

[15] R. G. Cole and J. H. Rosenbluth, "Voice over ip performance monitoring," ACM SIGCOMM Computer Communication Review, vol. 31, no. 2, pp. 9-24, 2001.

[16] R. Salami, C. Laflamme, B. Bessette, and J.-P. Adoul, "Itu-t g. 729 annex a: reduced complexity $8 \mathrm{~kb} / \mathrm{s}$ cs-acelp codec for digital simultaneous voice and data," IEEE Communications Magazine, vol. 35, no. 9, pp. 56-63, 1997. 